\title{
Practical challenges of managing COVID-19 patients in the adult intensive care unit of a tertiary care teaching hospital of Ireland
}

\author{
Shankar Lal ${ }^{1}$, Ehtesham Khan ${ }^{2}$, Muhammad Anwar Malik ${ }^{3}$ \\ 1-Registrar Anesthesiologist, Royal College of Surgeons Hospital Group, Republic of Ireland. \\ 2-Vice President, College of Anesthetists Ireland,Consultant Anesthesiologist, Royal College of Surgeons Hospital Group, Republic \\ of Ireland. \\ 3-Consultant Anesthesiologist, Royal College of Surgeons Hospital Group, Republic of Ireland.
}

Correspondence: Shankar Lal, Registrar Anesthesiologist, Royal College of Surgeons Hospital Group, Republic of Ireland; Mobile: 0879300866; E-mail: shankar.anaesthesia1@gmail.com

\begin{abstract}
The COVID-19 pandemic has attracted our focus to those important aspects of health care facilities in which we were severely lacking. Due to the unprecendeted nature of this pandemic, even the more developed nations of the world were not prepared to handle the challenges it presented. The current surge of critical patients of COVID-19 has led to a severe burden on our acute and critical care services, resulting in a significant increase in the demand and supply of hospital staff and equipment. There has been such pandemics in the past and they may occur again in future. Thus, to handle the current challenges, several measures must be adopted to handle the surge in confirmed critical cases, such as: expanding hospital capacity of critical area by converting noncritical area to critical areas, training and deployment of non-critical care staff to participate in delivering critical care brainstorming and implementing innovative approaches to obtain, conserve, and increase the efficiency of our available supply of physical equipment.
\end{abstract}

Key words: COVID-19; Pandemic; Ventilation; Critical care; Critical care unit

Citation: Lal S, Khan E, Malik M. Practical challenges of managing COVID-19 patients in the adult intensive care unit of a tertiary care teaching hospital of Ireland. Anaesth. pain \& intensive care 2020;24(3):263-271.

Received: 4 June 2020; Reviewed: 5 June 2020; Accepted; 7 June 2020

\section{Introduction}

On March 11th, 2020, the WHO (World Health Organization) announced the coronavirus disease (a respiratory tract infection) as a pandemic. It originally outbroke in Wuhan (China) and spread all around the world. It is caused by the beta coronavirus ${ }^{1}$ (Zoonotic Virus), a member of the severe acute metastasis syndrome-related coronavirus species (SARS-CoV-2) 2,3. COVID-19 ranges in severity from asymptomatic or moderate to severe; a considerable percentage of patients develop a more severe disorder ${ }^{1}$, depending on their co-morbidities.

The mortality rate amongst COVID-19 positive patients is usually stated to be about $2 \%$ to $3 \%$ but varies by country, patient age and comorbidities ${ }^{3}$. Due to the mutating nature of COVID-19 what we know is still rudimentary and needs ongoing research to enhance the understanding of its pathological pathway to develop advanced clinical management and medicine. COVID-19 may start with mild signs and symptoms and may progress to the point of respiratory distress and shock ${ }^{4}$. The most frequent complaints are fever (almost universal) and cough, which may or may not be productive. Myalgia and fatigue are also seen frequently. It results in hemoptysis, pleuritic chest pain in those suffering a severe form of $\mathrm{it}^{4}$, upper respiratory tract symptoms (rhinorrhea, sneezing, sore throat) are uncommon along with a headache.

Some patients have reported nausea, vomiting, and diarrhea. Patients should start self-isolation on the evidence of these signs and symptoms. Transmission 
of SARS-CoV-2 has been documented by Person-toperson $^{5}$ and is likely to happen by social contact ${ }^{6}$ and air droplets. ${ }^{7}$ Community transmission depicts that the transmission of the virus is possible even before the development of signs and symptoms. Transmission of SARS-CoV-2 by contact with contaminated environmental surfaces, fecal-oral have also been documented $^{6}$. Incidence and severity of COVID-19 have been seen more in older as compared to young. Males are affected relatively more than females, but this is still under research. It has not been so severe in teens and children, but they may have the asymptomatic carrier of the disease. COVID-19 should be suspected in those who have a travel history to highrisk areas. The nasopharyngeal swab should be taken in symptomatic and highly suspected patients, chest imaging should be done to record the presence of respiratory disease and to ascertain severity; if needed CT scan can be done. ${ }^{7}$ Clinical parameters are also very important peripheral saturation (pulse oximetry), respiratory rate, patient's breathing pattern and use of accessory muscles of respiration can help to understand the severity of the disease. ${ }^{7}$

\section{Practical Challenges of managing COVID-19 patients}

\subsection{Attending a suspected or confirmed COVID-19 patients in A\&E / Ward:}

\subsubsection{Risk of self-contamination on contact with patient or environment}

Always wear proper Personal Protective Equipment (PPE) (image1) before attending a suspected or confirmed case of COVID-19. There is no emergency in a pandemic, the safety of staff should always be sought. Institutions should make sure that all staff attending critical care areas are trained in proper donning and doffing of PPE.

\subsubsection{Risk of contamination of emergency drugs and intubation equipment.}

Emergency drugs and intubation equipment should be checked and kept outside the room. The runner should be available to provide drugs and intubation equipment at the door of the room in case of an emergency. Most often the runner is not from anesthesia department, it is the responsibility of primary anesthesiologist to inform the runner about drugs and equipment properly before going inside the room.

\subsection{Proning in ward}

We observed that the patients in the ward who received oxygen by venturi mask, high flow or CPAP improved once they were proned. Their PO2 improved and oxygen requirement went down and due to proning, some patients avoided intubation and ventilation. Proning these patients in the ward can be very helpful in situations where ventilators are not available, while also helping towards flattening the curve. ${ }^{8}$

\subsection{Airway Management and Ventilation \\ 2.3.1. Environment contamination with aerosol generation}

Patients showing severe signs and symptoms of COVID-19 often visit the A\&E department on high flow oxygen. There is a high risk of environmental contamination with aerosol generation in bearded, agitated and uncooperative patients.

It is recommended to keep these patients in isolation or in a single room with negative air pressure and a minimum of 6-12 air changes per hour. The door to the room must remain closed, except for when entering and exiting the room. If required, patients can be covered with transparent sheets to minimize aerosol contamination. Avoid nebulization, as it increases the risk of aerosol contamination. Proper ventilation (NIV) i.e., CPAP/BiPAP should be sought at the earliest. NIV should be applied with the most minimal pressure possible. There is no recommendation to use HFNO (Optiflow) as it increases the risk of aerosol generation and environmental contamination. If endotracheal intubation is needed, avoid panic, as team coordination is very important for the safety of the patient and staff. The anesthesiologist is the leader and is responsible for ensuring that intubation and eFONA (front of neck access) equipment is available and readily accessible. Avoid Bag mask ventilation and after proper preoxygenation of patient breathing spontaneously, give anesthesia drugs with a full dose of suxamethonium or rocuronium (if available) followed by intubation with an endotracheal tube of the highest possible internal diameter with video-laryngoscope. Inflate cuff of ETT, connect ventilator and check ventilation. Avoid Bag mask ventilation to check equal entry of air. During 


\section{Putting on PPE}

1. Decontaminate hands

2. Put on disposable apron/gown

3. Put on mask (Surgical or FFP2 or FFP3)

For FFP2 or FFP3 masks:

\begin{tabular}{l|ll}
\hline Fit Check & A. & Place mask over nose, mouth and chin \\
B. & Fit flexible nose piece over nose bridge \\
C. & Secure on head with elastic \\
D. & Adjust to fit \\
E. Inhale - mask should collapse \\
F. & Exhale - check for leakage around face \\
\hline
\end{tabular}

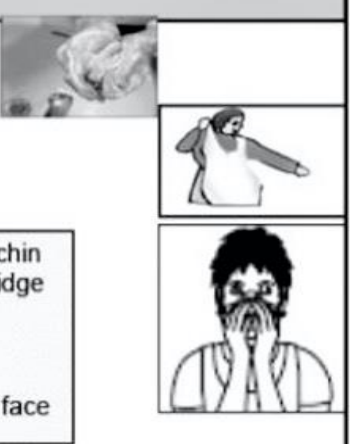

4. Put on goggles if required

5. Put on gloves

\section{Removing PPE}

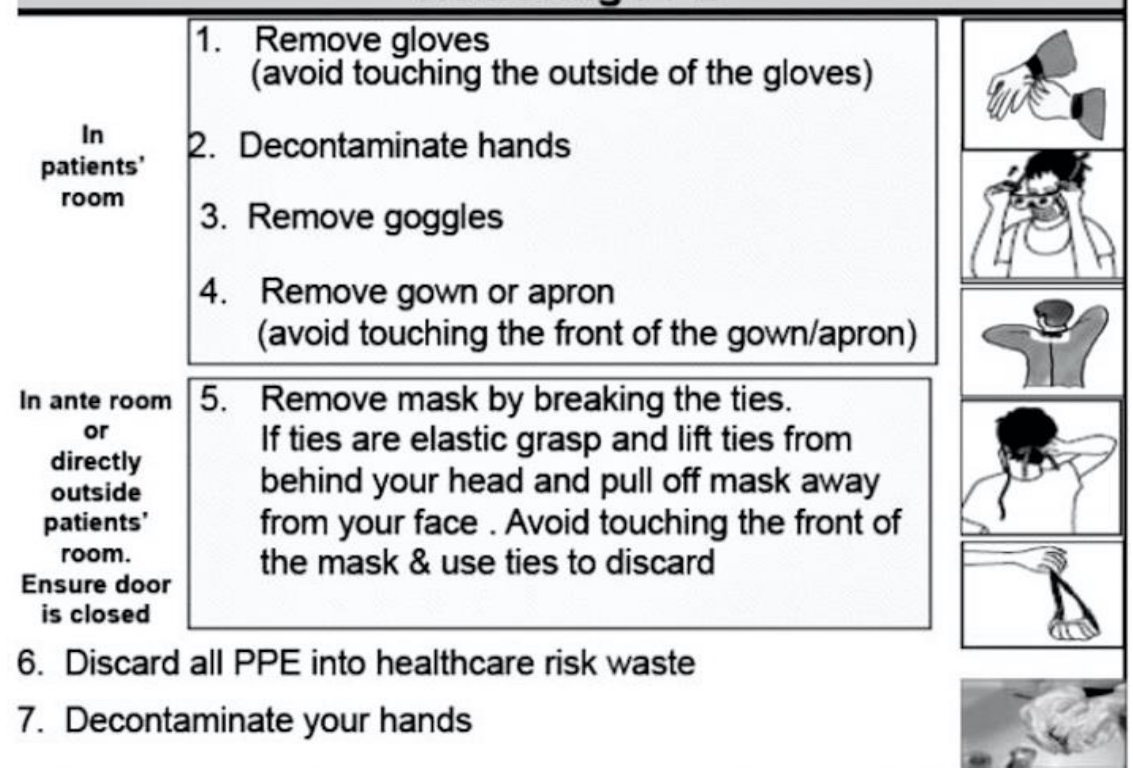

Figure 1: Correct sequence of putting on and removing PPE to prevent contamination of the face, mucous membranes and the clothes

pre-oxygenation/preparation of intubation, the patient can be covered with a transparent sheet (Image 2) or special intubation box (Image 3).

\subsection{Transferring a COVID-19 patient from $A \& E /$ ward to Isolation in ward/ICU.}

\subsubsection{Environment contamination and monitoring:}

There is always a great risk of aerosol generation and environmental contamination while transferring patients with COVID-19 disease.
The patient must wear a surgical mask during transport if they are getting high flow oxygen with a non-breathing mask/simple mask. The patient must be covered with a transparent sheet to minimize contamination. Escort the patient, if needed, to ensure the patient does not remove his/her mask during transport. Intubated patients should be transferred on portable ventilators after ensuring proper tubing and oxygen connections, and after making sure there is an adequate availability of oxygen in $E$ cylinder. The transporter must wear a gown, gloves, and mask when transporting patients on airborne precautions. The transporting unit must notify the receiving department of the necessary precautions, to reduce the risk of transmission of the infection.

The use of high-flow nasal oxygenation and non-invasive ventilation should be avoided due to an increased risk of aerosol generation. Due to the risk of coughing and aerosols, avoid awake fiberoptic intubation. To minimize the risk of contamination through respiratory aerosols, video laryngoscopy should be used. Patients usually have a very high alveolar-arterial gradient and are unable to tolerate a short period of apnea. Only small tidal volumes should be used in the case of manual ventilation. Rapid sequence induction (RSI) may be required. Five minutes of preoxygenation with $100 \%$ oxygen along with rapid sequence induction is required to avoid potential aerosolization of infectious respiratory droplets. Make sure a hydrophobic filter is placed between the breathing circuits and the facemask. Bagging should be avoided after intubation and the ventilator should be connected to an 


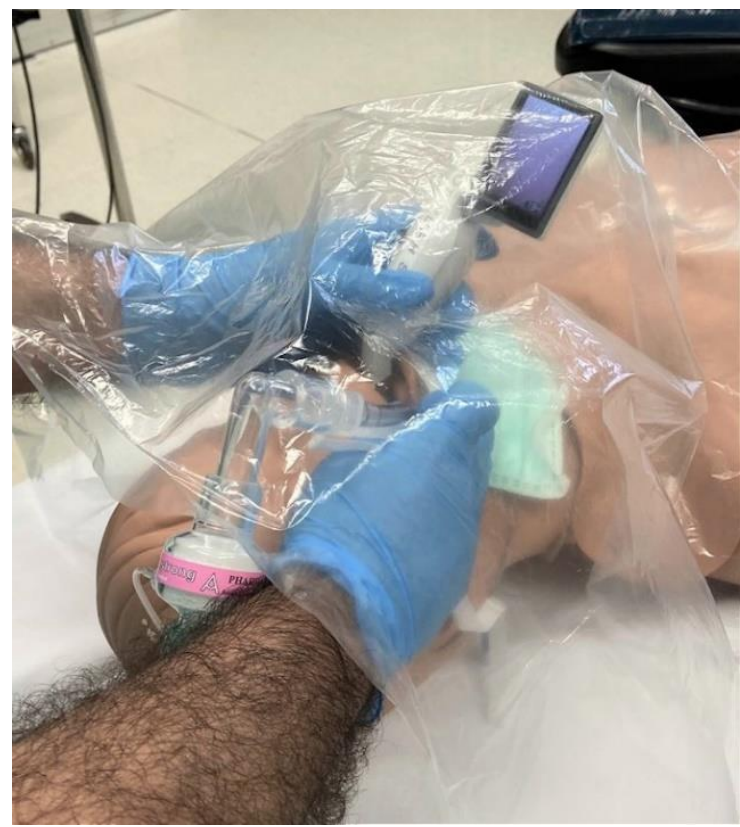

Image 2: Use of a transparent polythene sheet to prevent aerosols during intubation

endotracheal tube to confirm the correct position of the tube, with the help of end tidal carbon dioxide.

For improving ventilation and oxygenation, ARDS-net protocols for lung-protective strategy should be followed. For refractory hypoxemia, extracorporeal membrane oxygenation (ECMO) is an option if available. The role of pharmacological agents i.e. glucocorticoids, surfactant, salbutamol, nacetylcysteine, neutrophil esterase inhibitor, granulocyte-macrophage colony-stimulating factor can be used as per local guidelines.

\subsubsection{For decreasing airway secretions}

I.V glycopyrrolate can be used and intravenous salbutamol should be prepared for bronchospasm. Salbutamol and ipratropium nebulisation through the circuit should not be used due to an increased risk of contamination. We recommend against the use of nacetylcysteine and hypertonic nebulisations as both may lead to bronchospasm in patients of COVID-19.

\subsection{Proning the COVID-19 patient with severe ARDS}

Prone positioning improves oxygenation in most cases and promotes a more homogeneous distribution of ventilation, reducing the risk of ventilation induced lung injury. You will come across multiple challenges during and following proning the patients with severe

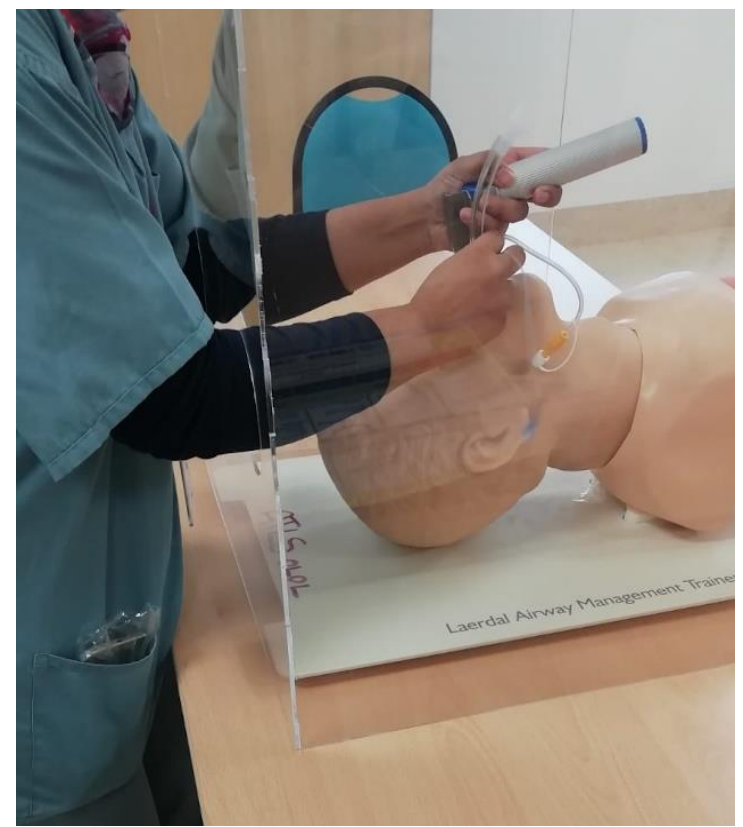

Image 3: Use of a transparent box to prevent aerosols during intubation

ARDS. Although the advantage of proning on improving oxygenation and patient survival is still debatable, it is frequently practiced in several health centers. In our centre we start proning if the patient's $\mathrm{P} / \mathrm{F}$ ratio is less than $<100 \mathrm{mmgh} /<13.3 \mathrm{kPa}$.

\subsection{Airway complications with prone position}

ETT kinking, displacement, dislodgement and disconnection. Circuit disconnection at elbow angle at the patient's end and connecting hoses at ventilator, airway trauma, de-saturation, bucking/coughing and gagging. ${ }^{9,10}$

Rule out contraindications for proning. Not all patients benefit from proning thus an appropriate selection of patients for proning should be sought. Proning sessions should be limited to 16 hours with a break of 8 hours by turning the patient to a supine position. Proning should be done with staff trained in proning patients. Check connections at patient and ventilator end, turn ventilator to $100 \%$ Fio2, do oral suctioning to avoid contamination and possible slippage of ETT, check cuff of ETT tube and give a full dose of muscle relaxants. Team dynamics are of utmost importance. Check airway pressure, tidal volume, oxygen saturation, and capnograph after proning the patient. 


\subsection{Development of Pressure ulcerations after prone position}

Significant numbers of patients have been observed suffering pressure ulceration to the forehead, mouth, nose, eyes, chest (breast in females), abdomen and pelvic organs (especially in a male). Face ulcers are mostly related to position and compression due to ETT, nasogastric tube, lines of invasive monitoring, ECG leads and tubes of intravenous infusions ${ }^{13,14}$.

Proper checks before and after turning the patient to the prone position can decrease/prevent complications. Head cushions or gel can decrease pressure ulceration on the face. We recommend the use of a surgical pillow (image $4 \mathrm{~A}$ and B) as it can help decrease airway trauma on ulceration along with the omission of frequent head turnings. Proper use of the ETT tie is also very valuable. Checking the tie tightness allows the passage of two fingers between the tie and each cheek. Use chest and abdomen cushions to decrease chest/breast, abdomen and pelvis compressions and ulcers.

\subsection{Neck strain/injury and nerve compression}

There are case reports reporting neck injury/strain and brachial plexus nerve injury due to extensive flexion and extension at the neck.

Proper checks before and after proning are very important and team dynamics are key. Avoid excessive compression and extension at neck, shoulder and elbow joints.

\subsection{Intracranial/intraocular intragastric pressure} and

Proning may result in a rise in intracranial/intraocular and intragastric pressure (present as intolerance to enteral feeding),

Rise in intracranial and intraocular can be ameliorated in a nursing patient in a reverse Trendelenburg position ${ }^{12}$. Intragastric pressure rise can be avoided by putting chest and pelvic support to decrease abdomen compression.

\subsection{Hemodynamic instability and arrhythmias}

Hypotension is common in prone position and there are case reports of cardiac arrhythmias and cardiac arrest occuring as well ${ }^{11}$.

Management of cardiovascular support is a great challenge in such patients. Careful attention to intravascular volume status should be given aiming conservative fluid strategy, with frequent monitoring of electrolytes, urine output, ABGs, BNP. Wherever needed, a vasopressor should be preferred to raise the blood pressures as compared to fluid boluses. Invasive monitoring is compulsory. Coagulation abnormalities should be corrected using blood products if necessary.

We noted the return of sinus rhythm from atrial fibrillation on turning the patient supine. Add endotracheal tube displacement in differential diagnosis while managing atrial fibrillation.

\subsection{Number of staff required and risk of injury to staff}

Proning requires a great team dynamic. It requires 7 people, one at the head end (anesthesiologist/intensivist) and three on each side of the patient. It is hard to prone patients with a high BMI as there are reported cases of various degrees of injury to staff.

Meticulous care should be taken during proning. Only staff trained in proning should be the part of the team, as a strong team dynamic is key.

\section{Circulatory Support}

We noticed frequent episodes of bradycardia, tachycardia, atrial fibrillation and supraventricular tachycardia in COVID-19 positive patients.

Arrythmias in COVID-19 positive patients may be multifactorial; we have evidenced myocarditis as one of the causes of arrythmias.

\section{Renal Support}

Acute kidney injury is common in severe cases. There are multiple factors responsible for AKI in such patients e.g., Hypoxic Kidney Injury, hypo-perfusion, raised CK levels, viral load, and generation of superoxides.

We recommend the re-checking of urinary Foley's catheter placement and position as there are reported cases of anuria due to kinking and compression of Foley's catheter in prone position. Adequate (judicious) pre-renal volume and avoidance of abdominal pressure should be considered as well. The role of the early start of CRRT has not been established 
yet, but whenever needed, escalation in therapy should be done as per local and management policies.

\section{Antibiotics and experimental therapies}

No definite treatment is available.

Some centers have reported benefits with the use of a combination of retroviral medicine with hydroxychloroquine and azithromycin. Patients having high QTc are not suitable for use of Hydrochloquine and azithromycin as both can cause fatal arrhythmias and cardiovascular shock in such patients.

Patients having high aspirated are also unable to take these medications.

We noted the melting of a nasogastric tube made of polyurethane on the administration of retroviral lopinavir-ritonavir (Kaletra) medicine. We found NG tubes made up of PVC suitable for the administration of Kaletra. Patients with evidence of septic shock and secondary infection should be treated with broadspectrum antimicrobials.

The development of immunity against COVID-19 in patients recovering from the disease is still not fully evidenced. There are some case reports from different countries where convalescent plasma is being used to treat symptomatic COVID-19 patients, and significant recovery has been reported in those cases. ${ }^{16}$

\section{Resistant Hyperpyrexia}

COVID-19 patients present with persistent hyperpyrexia unresponsive to regular medications. It may be multifactorial.

Recommendation: Fever is one of the common symptoms in symptomatic COVID-19 patients as it is one of the most important physiological adoptive responses to infection. Always rule out the cause of pyrexia in relation to the patient's clinical condition, history, investigations and symptom pattern. Superimposed infection may be the cause of pyrexia in COVID-19 patients.

Although there is very limited data available on this, according to some observational studies, fever is a good prognostic sign in critically ill patients. It inhibits microbial re-production and viral replication along with enhancing the rate of phagocytosis. It also has an additional benefit of inhibiting pro-inflammatory signaling pathway via heat-shock proteins. ${ }^{15}$

\section{Extubation \& post- extubation care}

Extubation of confirmed COVID-19 patients is an enormous challenge. Coughing, bronchospasm, disconnections, ETT biting, kinking and self extubation are some of the examples, and along with it, care of a extubated covid-19 positive patient in ICU/ HDU is also a big challenge.

Before considering any patient for extubation, make sure each member is with proper PPE, and individual roles have been assigned in case re-intubation is needed. Review that the patient is fully ready and optimised for extubation because extubation and reintubation are both high aerosol-generating procedures that may increase the risk of environmental contamination and exposure to staff. We recommend that you follow your local or national weaning protocols and make sure the patient has regular breathing and optimum oxygenation on pressure support/SIMV mode of ventilation. We use a P/F ratio of more than $250 \mathrm{mmHg}(>33.3 \mathrm{kPa})$ with regular, spontaneous breathing as one of our criteria for extubation, along with a stable hemodynamic with minimum vasopressor / inotropic support. We urge you to blunt the cough response completely by using different medications or by following your local guidelines. We use boluses of IV lignocaine before and following extubation, to blunt the cough response.

Following extubation, the patient should be continuously monitored in the negative pressure room with $\mathrm{ABG}$ and chest $\mathrm{x}$-rays as per need. Patient transfer to step down should be done following local guidelines in a ward where there is facility of negative pressure rooms.

\section{Tracheostomy of COVID-19 patient}

Tracheostomy is systematically recognized as an aerosol-generating procedure ${ }^{17}$. Therefore, it is advisable to consider it a high-risk procedure for both the staff performing the tracheostomy and for the staff 
who will be doing nursing care of the patient afterwards. This risk may even be increased due to the frequent need to suction through tracheostomy and the need to clean and change the inner tube, along with the risk of tracheostomy tube displacement during a severe cough and position changes. Other problems include the anticipated need to use large volumes of PPE for all staff, with meticulous donning and doffing procedures due to the increased risk of aerosolization. There may be an increased risk of tracheostomy complications since patients may not be treated by experienced/trained staff in the case of a pandemic.

Recommendation: Delay both open and percutaneous tracheostomy. Based on the above risks, it is recommended that tracheostomies should not be carried out routinely, to allow time for reduction in viral load in patients with COVID-19. The decision to intervene should be promptly evaluated by the most experienced surgeon available, in close consultation with the anesthesiologist, considering the availability of resources and local or national guidelines. Always consider patients as positive for COVID-19 and approach them with complete PPE. Procedures should only be done in the ICU or operating rooms with negative pressure and with designated routes within the operating theatre complex for patient transportation. Make sure adequate doses of muscle relaxants are used during the procedure. If it is unavoidable to proceed with the tracheostomy, percutaneous tracheostomy should be encouraged over open/surgical tracheostomy because of a decreased risk of aerosolization. During the transfer of the patient from the ICU to the ward, make sure all staff is wearing proper PPE, and that the patient is covered with a transparent sheet with the minimum possible oxygen. Handover the patient to a minimum number of staff in the ward to decrease the exposure.

\section{Workload and staff well- being}

Due to the rising number of COVID-19 positive patients requiring admission to the intensive care unit, the Critical care staff workload has significantly increased. The high risk of self-contamination and fear of transmission of infection to others, especially family members, has resulted in enhanced emotional stress among health care workers.
Considering the work overload and metal stress, working hours can be split into feasible shifts with frequent rotations, to less stressful duties on a weekly basis, along with minimizing the number of night duties as suitable. Individual meetings with small groups of anesthesiologists must be practiced, especially with more vulnerable staff. Staff well-being and mental health are very important. Mentally and emotionally occupied staff frequently make a mistakes which can be dangerous to themselves, patients and/or other healthcare staff.

\section{Ethical issues and escalation}

Treatment of a patient suffering from a severe form of COVID-19 disease requires extraordinary resources.

The patients with a higher clinical likelihood of benefit, should be given precedence. This decision should be taken on an individual basis after a multidisciplinary team decision and joint agreement. We are using our local critical care triage guidelines for admitting COVID-19 positive or highly suspected patients where we calculate the patient's frailty score, SOFA score along with their co-morbidities and wish for escalation if the patient is oriented. Taking into the consideration the high mortality of severe cases, secondary contamination to health care staff and accessibility of resources can be justified. This may help decrease demand on the possible restriction of surgeries, the transformation of post anesthesia care units and level 1 and 2 beds to level 3 along with rapid retraining and redeployment of clinical and nursing staff that may influence the hospital's ability to staff other administrations.

We increased the capacity of our intensive care unit from 10 beds to 25 beds by purchasing new transport ventilators. We took the anesthesia machines from our theatres induction room and set them into the post anesthesia care unit and operating rooms. To make their use safer and more effective, we added HME filters on inspiratory and expiratory hoses of anesthesia machines.

\section{Summary}

Due to the unprecendeted nature of Covid 19 and to meet the current challenges, several measures must be 
adopted to handle the surge in confirmed critical cases, such as:

Expanding hospital capacity of critical area by converting non-critical area to critical areas.

Training and deployment of non-critical care staff to participate in delivering critical care

Brainstorming and implementing innovative approaches to obtain, conserve, and increase the efficiency of our available supply of physical equipment.

\section{Conflict of interest}

No conflict of interest is declared by the authors.

\section{Authors' contribution}

All authors took part in the preparation of this manuscript.

\section{References}

1. Phelan AL, Katz R, Gostin LO. The novel coronavirus originating in Wuhan, China: challenges for global health governance. $2020 . \quad$ DAMA. 10.1001/jama.2020.1097

2. Gorbalenya AE, Baker SC, Baric RS, de Groot RJ, Drosten $C$, Gulyaeva AA, et al. Severe acute respiratory syndrome-related coronavirus: the species and its viruses-a statement of the coronavirus study group. bioRxiv. 2020. DOI: 10.1101/2020.02.07.937862

3. Chen N, Zhou M, Dong X, Qu J, Gong F, Han Y, et al. Epidemiological and clinical characteristics of 99 cases of 2019 novel coronavirus pneumonia in Wuhan, China: a descriptive study. Lancet. 2020;395(10223):507-513. DOI: $\underline{10.1016 / S 0140-6736(20) 30211-7}$

4. Yang X, Yu Y, Xu J, Shu H, Xia J, Liu H, et al. Clinical course and outcomes of critically ill patients with SARSCoV-2 pneumonia in Wuhan, China: a single-centered, retrospective, observational study. Lancet Respir Med. 2020 May;8(5):475-481. [PubMed] DOI: $10.1016 / \mathrm{S} 2213-2600(20) 30079-5$

5. Chan JW, Ng CK, Chan YH, Mok TY, Lee S, Chu SY, et al. Short term outcome and risk factors for adverse clinical outcomes in adults with severe acute respiratory syndrome (SARS). Thorax. 2003 Aug;58(8):686-689. [PubMed] DOI: $10.1136 /$ thorax.58.8.686

6. Li Q, Guan X, Wu P, Wang X, Zhou L, Tong Y, et al. Early transmission dynamics in Wuhan, China, of novel coronavirus-infected pneumonia. N Engl J Med. 2020
Mar 26;382(13):1199-1207. [PubMed] DOI: 10.1056/NEJMoa2001316

7. Kalil AC, Metersky ML, Klompas M, Muscedere J, Sweeney DA, Palmer LB, et al. Management of adults with hospital-acquired and ventilator-associated pneumonia: 2016 clinical practice guidelines by the infectious diseases society of america and the american thoracic society. Clin Infect Dis. 2016;63:e61-111. DOI: $10.1093 / \mathrm{cid} / \mathrm{ciw} 353$

8. Sun $Q$, Qu $H$, Huang M, Yang Y. Lower mortality of COVID-19 by early recognition and intervention: experience from Jiangsu Province. Ann Intensive Care. 2020 Mar 18;10(1):33. [PubMed] DOI: 10.1186/s13613020-00650-2

9. Lee JM, Bae W, Lee YJ, Cho YJ. The efficacy and safety of prone positional ventilation in acute respiratory distress syndrome: updated study-level meta-analysis of 11 randomized controlled trials. Crit Care Med. 2014:42(5):1252-1262. [PubMed] DOI: 10.1097/CCM.0000000000000122

10. Hu SL, He HL, Pan C, Liu AR, Liu SQ, Liu L, et al. The effect of prone positioning on mortality in patients with acute respiratory distress syndrome: a meta-analysis of randomized controlled trials. Crit Care. 2014;18(3):R109. [PubMed] DOI: $\underline{10.1186 / \mathrm{cc} 13896}$

11. Hu SL, He HL, Pan C, Liu AR, Liu SQ, Liu L, et al. The effect of prone positioning on mortality in patients with acute respiratory distress syndrome: a meta-analysis of randomized controlled trials. Crit Care. 2014;18(3):R109. [PubMed] DOI: 10.1186/cc13896

12. DeJong A, Molinari N, Sebbane M, Prades A, Futier E, Jung $B$, et al. Feasibility and effectiveness of prone position in morbidly obese patients with ARDS. Chest. 2013;143(6):1554-1561. [PubMed] DOI: 10.1378/chest.12-2115

13. Jolliet $P$, Bulpa $P$, Chevrolet JC. Effect of the prone position on gas exchange and hemodynamics in severe acute respiratory distress syndrome. Crit Care Med. 1998;26(12):1977-1985. [PubMed] DOI: 10.1097/00003246-199812000-00023

14. Brüssel T, Hachenberg T, Roos N, Lemzem H, Konertz $W$, Lawin P. Mechanical ventilation in the prone position for acute respiratory failure after cardiac surgery. J Cardiothorac Vasc Anesth. 1993;7(5):541546. [PubMed] DOI: 10.1016/1053-0770(93)90311-8

15. Swenson BR, Hedrick TL, Popovsky K, Pruett TL, Sawyer RG. Is fever protective in surgical patients with bloodstream infection? J Am Coll Surg. 2007 May;204(5):815-821. [PubMed] DOI: 10.1016/j.jamcollsurg.2007.01.033

16. Shen C, Wang Z, Zhao F, Yang Y, Li J, Yuan J, et al. Treatment of 5 critically ill patients with COVID-19 
with convalescent plasma. JAMA. 2020 Mar 27;323(16):1582-1589. [PubMed] DOI: 10.1001/jama.2020.4783

17. HSE Health Protection Surveillance Centre. Guidance on covid-19 v2.0, 23.03.2020. Use of PPE to support infection prevention and control practice when performing aerosol generating procedures on confirmed or clinically suspected covid-19 cases in a pandemic situation. Available from: https://acslm.ie/wpcontent/uploads/2020/04/AGPs-for-confirmed-orpossible-COVID19_v2.0_23032020.pdf 